
\title{
$\begin{array}{ll}\text { Research Square } & \begin{array}{l}\text { Preprints are preliminary reports that have not undergone peer review. } \\ \text { They should not be considered conclusive, used to inform clinical practice, } \\ \text { or referenced by the media as validated information. }\end{array}\end{array}$
}

\section{Association between Serum Vitamin A Levels and Premature Ovarian Insufficiency Risk}

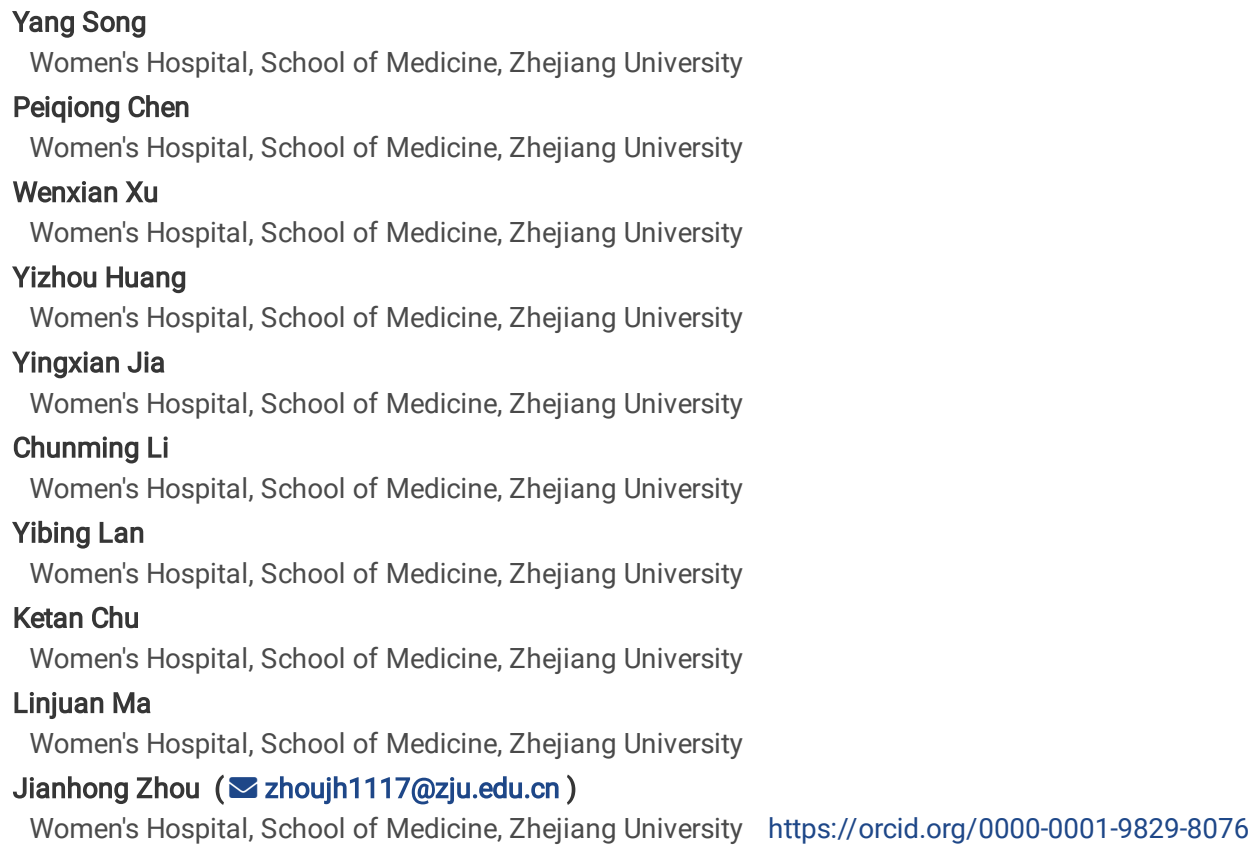

\section{Research Article}

Keywords: vitamin A, premature ovarian insufficiency, anti-Müllerian hormone

Posted Date: February 15th, 2021

DOI: https://doi.org/10.21203/rs.3.rs-192703/v1

License: (a) (1) This work is licensed under a Creative Commons Attribution 4.0 International License. Read Full License 


\section{Abstract}

Purpose: The aim of the study was to explore the association between serum vitamin A levels and premature ovarian insufficiency (POI).

Methods: In this cross-sectional survey, women with POI $(n=47)$ and normo-ovulatory controls $(n=67)$ were enrolled from December 2016 to May 2018 in Zhejiang, China. The serum levels of follicle-stimulating hormone ( $\mathrm{FSH})$, luteinizing hormone ( $\mathrm{LH})$, estradiol ( $\left.\mathrm{E}_{2}\right)$, anti-Müllerian hormone (AMH), vitamin $\mathrm{A}$, and total cholesterol (TC) were measured for each participant. The association of vitamin A levels with POI risk was assessed using binary logistic regression analysis.

Results: Serum vitamin A levels seems slightly higher in the POI group than in the control group (728.00 $\pm 176.00 \mathrm{vs} 503.93 \pm 145.64 \mu \mathrm{g} / \mathrm{L}, p=0.133)$. But after adjust with serum lipid level, serum vitamin A/TC ratio was significantly lower in the POI group than in the control group (143.14 \pm 35.86 vs $157.56 \pm$ $35.21 \mu \mathrm{g} / \mathrm{mmol}, p=0.035)$. Further, serum vitamin A/TC ratio was significantly and negatively associated with POI risk [unadjusted odds ratio (OR) $=0.988$, $95 \%$ confidence interval $(\mathrm{Cl}): 0.977-0.999, p=0.039]$. The same trend was found after adjusting for confounding factors (age, BMI, annual household income, and education) $(\mathrm{OR}=0.986,95 \% \mathrm{Cl}: 0.972-0.999, p=0.040)$.

Conclusion: Serum vitamin A/TC ratio was negatively associated with POI risk, indicating that vitamin A deficiency may be a risk factor for POI development. Serum vitamin A/TC ratio may serve as a predictive factor for $\mathrm{POI}$ incidence. Thus, vitamin A may serve a protective role in ovarian tissue.

\section{Introduction}

Premature ovarian insufficiency (POI) currently affects approximately $1 \%$ of the female population [1]. Women with POI suffer from ovarian dysfunction and infertility, and POI is also associated with an increased risk of mortality, cancer, cardiovascular disease, and osteoporosis [2-3]. Although ovarian insufficiency has drawn much concern over the past few decades, the etiology of approximately $90 \%$ of POI cases remains unknown [4]. POI may be correlated with chromosomal abnormalities, environmental pollutants, social stress, childbearing postponement, autoimmunity, and prolonged survival of patients after gonadotoxic treatments such as with paclitaxel $[1,5-6]$. Therefore, studies on the etiology of POI are warranted.

Vitamin A (retinol), a fat-soluble vitamin, is essential for reproduction, deficiencies or excesses may result in embryonic loss and/or congenital defects. [7-9] Studies have shown that the vitamin A signaling pathway has potential effects on follicular development, oocyte maturation, ovarian steroidogenesis, and corpus luteum formation [10-12]. Also, studies showed that vitamin A level in follicular fluid had an association with the quality of human oocytes, fertilization potential and embryo development[13]. However, Vitamin A deficiency (VAD) is common in women of reproductive age and even more serious in pregnant women. In low-income countries, $10-20 \%$ of the pregnant women develop VAD [14]. Serum vitamin A levels in women with POI have seldom been considered before.

Lately, many studies have confirmed the role of vitamin A in ovarian physiological function. To explore the association between serum vitamin A levels and $\mathrm{POI}$ risk in women of reproductive age, we conducted a case-control cross-sectional study of young women diagnosed with POI and normo-ovulatory controls matched by age and body mass index (BMI). To the best of our knowledge, this is the first study to report the association between vitamin A levels and POI risk in women of reproductive age.

\section{Materials And Methods Study Population}

This study was performed in the Department of Gynecology at Women's Hospital, School of Medicine, Zhejiang University, Hangzhou, Zhejiang, China. A total of 47 patients identified as having POI between December 2014 and May 2018 were identified from laboratory records and medical history [age, mean (range): $33.30 \pm 6.45$ (18-42) years], 8 women above 40 years old at enrollment was diagnosed POI before 40 years old. The control group, from health examination, included 67 women of reproductive age [age, mean (range): $32.63 \pm 5.87(21-43)$ years] with normal menstrual cycles. The study protocol was in accordance with the Declaration of Helsinki and was approved by Women's Hospital, School of Medicine, Zhejiang University's ethics committee. Informed consent was obtained from all participants.

\section{Definitions}

POI diagnosis was based on the European Society of Human Reproduction and Embryology (ESHRE) criteria [15]. The eligibility criteria for this study included oligomenorrhea or amenorrhea for at least 4 months, elevated follicle-stimulating hormone (FSH) levels $>25$ IU/L on two measurements more than 4 weeks apart, and age below 40 years at the first diagnosis. The control group was recruited from routine physical check-ups and matched with women with POI based on $\mathrm{BMI}$ and age $( \pm 3$ years $)$.

The exclusion criteria were as follows: patients with autoimmune diseases; chronic diseases (liver diseases, renal diseases, hypertension, cardiovascular or cerebrovascular diseases, or congestive heart failure); or history of hysterectomy, oophorectomy, chemotherapy, radiotherapy, or hormone replacement therapy. Additionally, patients who smoked, were using vitamin supplements, or were currently pregnant or breast-feeding were excluded.

Analysis was conducted after obtaining completed questionnaires from each participant. Trained interviewers conducted face-to-face interviews to obtain a detailed picture of the women's social demographics, gynecological medical history, lifestyle factors, and reproductive and medical history. Age, height, weight, occupation, education, and annual household income of each participant were recorded. Anthropometric measurements (body mass and height) were 
performed, and BMI was calculated according to the standard formula. Normal weight was defined as a BMl $=18.5-23.9 \mathrm{~kg} / \mathrm{m}^{2}$ and obesity as $\mathrm{BMI}>30.0$ $\mathrm{kg} / \mathrm{m}^{2}$.

\section{Blood Collection}

Peripheral blood was collected randomly at the time of the interview from women with POI. Venous blood was collected from controls during the early follicular phase of the menstrual cycle (days 1-5 of a spontaneous bleeding episode) to evaluate the basal levels of FSH, luteinizing hormone (LH), estradiol $\left(E_{2}\right)$, and anti-Müllerian hormone (AMH). All samples were collected and used according to the manufacturers' recommendations in kits. After obtaining venous blood samples, the plasma was separated by centrifugation for $10 \mathrm{~min}$ at $4000 \mathrm{rpm}$ at $4^{\circ} \mathrm{C}$; within 30 min, the samples were transferred into airtight vials and stored at $-80^{\circ} \mathrm{C}$. TC was measured enzymatically within 6 weeks of collection.

\section{Hormone Measurements}

Serum levels of FSH, LH, $E_{2}$, and AMH were determined using an automated Roche Modular Analytics E170 immunoassay system (Roche Diagnostics, Mannheim, Germany). Serum vitamin A levels were measured via reversed-phase high-performance liquid chromatography equipped with an ultraviolet detector and computerized data acquisition and storage (Pharmacia Biotech, Uppsala, Sweden). The samples were stored for $<1.5$ years and were not thawed prior to analysis.

Because plasma vitamin A levels change with the amount of synchronized lipids, lipid standardization is necessary to eliminate confounding effects. Jordan et al. [16] and Horwitt et al. [17] suggested standardization to the total lipids. Thurnham et al. [18] found that the sum of cholesterol is nearly as powerful as the adjustment for total lipids. To ensure that TC levels did not affect the results, vitamin A/TC ratio was calculated.

\section{Statistical Analysis}

Data processing and statistical analysis were performed using SPSS software (version 17; SPSS Inc., Chicago, IL, USA). Continuous variables were presented as mean and standard deviation (SD). The Kolmogorov-Smirnov test was applied to test the normality of the data distributions (all data were presented as mean \pm SD). Continuous variables with normal distributions were compared using Student's t-test. Continuous variables with non-normal distribution were compared using Mann-Whitney U test. Categorical variables were presented as number and percentage and compared using chi-square test. The correlations of each variable with POI were assessed with Spearman nonparametric correlation analysis. The association between the TC-adjusted vitamin A levels and $\mathrm{POI}$ risk was further assessed using binary logistic regression analysis. All statistics were two-tailed, and $p<0.05$ was considered significant.

\section{Results}

Demographic characteristics and biochemical parameters of all participants in this study are presented in Table 1. There were no significant differences in age and $\mathrm{BMI}$ at enrollment between the POI and control groups ( $p=0.514$ and $p=0.633$, respectively). Women in the control group showed a significantly higher annual household income and education than those in the POI group (both $p<0.001$ ). Serum FSH and LH levels in the POI group were significantly higher than those in the control group (72.32 \pm 32.84 vs $6.82 \pm 2.26 \mathrm{IU} / \mathrm{L}, p<0.001 ; 39.48 \pm 16.25$ vs $4.10 \pm 1.51 \mathrm{IU} / \mathrm{L}, p<0.001$, respectively). Conversely, serum AMH levels were lower in the POI group than in the control group $(0.02 \pm 0.02 \mathrm{vs} 3.36 \pm 2.37 \mathrm{ng} / \mathrm{mL}, p<0.001)$. Serum vitamin A levels seems slightly higher in the POI group than in the control group ( $728.00 \pm 176.00$ vs $503.93 \pm 145.64 \mu \mathrm{g} / \mathrm{L}, p=0.133)$. But after adjust with serum lipid level, serum vitamin $\mathrm{A} / \mathrm{TC}$ ratio was significantly lower in the POI group than in the control group (143.14 $\pm 35.86 \mathrm{vs} 157.56 \pm 35.21 \mu \mathrm{g} / \mathrm{mmol}, p=0.035)$. 
Table 1

Clinical characteristics and biochemical parameters of participants

\begin{tabular}{|c|c|c|c|}
\hline Variables & POI group $(n=47)$ & Control group $(n=67)$ & $p$-Value \\
\hline \multicolumn{4}{|l|}{ Age at enrollment, years (\%) } \\
\hline $18-25$ & $6(12.8)$ & $7(10.4)$ & \\
\hline $25-29$ & $7(14.9)$ & $15(22.4)$ & \\
\hline $30-34$ & $12(25.5)$ & $18(26.9)$ & \\
\hline $35-39$ & $14(29.8)$ & $17(25.4)$ & \\
\hline$>40$ & $8(17.0)$ & $10(14.9)$ & \\
\hline Mean \pm SD & $33.30 \pm 6.45$ & $32.63 \pm 5.87$ & $0.469^{a}$ \\
\hline \multicolumn{4}{|l|}{$\mathrm{BMl}, \mathrm{kg} / \mathrm{m}^{2}(\%)$} \\
\hline$<18.5$ & $6(12.8)$ & $13(19.4)$ & \\
\hline $18.5-23.9$ & $36(76.6)$ & $45(67.2)$ & \\
\hline $24.0-30$ & $5(10.6)$ & $9(13.4)$ & \\
\hline Mean \pm SD & $21.11 \pm 2.25$ & $20.88 \pm 2.64$ & $0.633^{b}$ \\
\hline \multicolumn{4}{|c|}{ Annual household income, RMB yuan (\%) } \\
\hline$\otimes 30000$ & $7(14.9)$ & $1(1.5)$ & $<0.001^{c}$ \\
\hline $30000-100000$ & $21(44.7)$ & $10(14.9)$ & \\
\hline$\otimes 100000$ & $19(40.4)$ & $56(83.6)$ & \\
\hline \multicolumn{4}{|l|}{ Education,n (\%) } \\
\hline Elementary school & $13(27.7)$ & $4(6.0)$ & $<0.001^{c}$ \\
\hline High school & $15(31.9)$ & $6(9.0)$ & \\
\hline College & $19(40.4)$ & $57(85.1)$ & \\
\hline \multicolumn{4}{|l|}{ Biochemical parameters } \\
\hline \multicolumn{4}{|l|}{ Mean \pm SD (Range) } \\
\hline FSH (IU/L) & $72.32 \pm 32.84(27.86-149.47)$ & $6.82 \pm 2.26(2.25-15.56)$ & $<0.001^{\mathrm{a}}$ \\
\hline LH (IU/L) & $39.48 \pm 16.25(14.33-77.56)$ & $4.10 \pm 1.51(1.13-8.08)$ & $<0.001^{\mathrm{b}}$ \\
\hline E2 (pmol/L) & $188.61 \pm 151.02(43.31-755.46)$ & $150.20 \pm 74.37(69.11-560.06)$ & $0.368^{\mathrm{a}}$ \\
\hline $\mathrm{AMH}(\mathrm{ng} / \mathrm{mL})$ & $0.02 \pm 0.02(0.01-0.08)$ & $3.36 \pm 2.37(0.18-11.28)$ & $<0.001^{a}$ \\
\hline Vitamin A (ug/L) & $728 \pm 176(349-1162)$ & $662 \pm 113(374-943)$ & $0.133^{\mathrm{b}}$ \\
\hline $\mathrm{TC}(\mathrm{mmol} / \mathrm{L})$ & $5.20 \pm 1.07(3.86-9.77)$ & $4.31 \pm 0.74(2.76-6.87)$ & $<0.001^{\mathrm{b}}$ \\
\hline Vitamin A /TC (ug/mmol) & $143.14 \pm 35.86(62.95-218.87)$ & $157.56 \pm 35.21(84.04-256.16)$ & $0.035^{b}$ \\
\hline \multicolumn{4}{|c|}{ Data are expressed as $n(\%)$ or mean \pm standard deviation; $p<0.05$ indicates statistical significance. } \\
\hline \multicolumn{4}{|c|}{$\begin{array}{l}\text { POI, premature ovarian insufficiency; BMI, body mass index; SD, standard deviation; FSH, follicle-stimulating hormone; LH, luteinizing hormone; E2, } \\
\text { estradiol; AMH, anti-Müllerian hormone. }\end{array}$} \\
\hline \multicolumn{4}{|l|}{ aMann-Whitney Utest } \\
\hline bStudent's t-test for indepen & & & \\
\hline
\end{tabular}

The POI group was divided into two subgroups based on serum FSH levels (Table 2). Serum vitamin A/TC levels were significant lower in the two POI groups than the control group. Meanwhile, Serum vitamin A/TC levels in the POI group with low FSH levels ( $<40$ IU/L) was lower than that in the POI group with high FSH levels ( $\geq 40 \mathrm{IU} / \mathrm{L}$ ), although the difference was not statistically significant (144.95 $\pm 39.40 \mathrm{vs} 142.77 \pm 35.64 \mu \mathrm{g} / \mathrm{mmol}, p>0.05)$. 
Table 2

Corrected Serum vitamin A levels in women with different FSH levels

\begin{tabular}{|llll|}
\hline & Control group & POI group & \\
\hline FSH(IU/L) & $<25(n=47)$ & $25-40(n=8)$ & $>40(n=39)$ \\
\hline & Mean \pm SD & Mean \pm SD & Mean \pm SD \\
\hline Vitamin A/TC (ug/mmol) & $157.56 \pm 35.21$ & $144.95 \pm 39.40$ & $142.77 \pm 35.64$ \\
\hline
\end{tabular}

Table 3 showed that annual household income, education, serum AMH, and serum vitamin A/TC ratio were significantly and negatively associated with POI risk $(p<0.05$ all) by spearman correlation analysis. However, the serum TC level significantly and positively associated with POI risk in the study population $(p$ $<0.05)$.

Table 3

Association of $\mathrm{POI}$ with confounders ${ }^{\mathrm{a}}$

\begin{tabular}{|c|c|c|c|c|c|c|c|c|c|c|c|c|}
\hline Confounders & POI & Age & BMI & $\begin{array}{l}\text { Annual } \\
\text { household } \\
\text { income }\end{array}$ & Education & $\begin{array}{l}\text { FSH } \\
(\mathrm{IU} / \mathrm{L})\end{array}$ & $\begin{array}{l}\text { LH } \\
\text { (IU/L) }\end{array}$ & $\begin{array}{l}\mathrm{E}_{2} \\
( \\
\mathrm{pmol} / \mathrm{L})\end{array}$ & $\begin{array}{l}\text { AMH } \\
(\mathrm{ng} / \mathrm{mL})\end{array}$ & $\begin{array}{l}\text { Vitamin } \\
\text { A } \\
\text { (ug/L) }\end{array}$ & $\begin{array}{l}\text { TC } \\
(\mathrm{mmol} / \mathrm{L})\end{array}$ & $\begin{array}{l}\text { Vitamin A } \\
\text { /TC } \\
\text { (ug/mmol) }\end{array}$ \\
\hline \multicolumn{13}{|l|}{ POI } \\
\hline Age & 0.061 & & & & & & & & & & & \\
\hline BMI & 0.101 & 0.079 & & & & & & & & & & \\
\hline $\begin{array}{l}\text { Annual } \\
\text { household } \\
\text { income }\end{array}$ & $-0.457^{\star \star}$ & 0.098 & -0.126 & & & & & & & & & \\
\hline Education & $-0.463^{\star \star}$ & -0.151 & -0.111 & $0.504^{*}$ & & & & & & & & \\
\hline $\mathrm{FSH}(\mathrm{IU} / \mathrm{L})$ & $0.853^{* \star}$ & $0.221^{*}$ & -0.042 & $-0.322^{\star *}$ & $-0.385^{\star \star}$ & & & & & & & \\
\hline LH(IU/L) & $0.853^{\star \star}$ & 0.113 & -0.038 & $-0.389^{* *}$ & $-0.388^{\star *}$ & $0.824^{* *}$ & & & & & & \\
\hline $\mathrm{E}_{2}(\mathrm{pmol} / \mathrm{L})$ & 0.085 & 0.080 & -0.083 & -0.115 & -0.005 & 0.037 & 0.000 & & & & & \\
\hline AMH(ng/mL) & $-0.864^{\star \star}$ & $-0.258^{\star *}$ & -0.12 & $0.328^{\star \star}$ & $0.400^{\star \star}$ & $-0.835^{\star \star}$ & $-0.708^{\star}$ & -0.142 & & & & \\
\hline $\begin{array}{l}\text { Vitamin A } \\
\text { (ug/L) }\end{array}$ & $0.207^{*}$ & 0.089 & $0.216^{*}$ & -0.142 & $-0.189^{*}$ & $0.617^{\star \star}$ & 0.136 & 0.018 & $-0.208^{*}$ & & & \\
\hline $\begin{array}{l}\text { TC } \\
(\mathrm{mmol} / \mathrm{L})\end{array}$ & $0.463^{\star \star}$ & $0.200^{\star}$ & 0.092 & $-0.186^{*}$ & $-0.260^{\star \star}$ & $0.399^{\star \star}$ & $0.423^{*}$ & 0.102 & $-0.355^{\star *}$ & $0.266^{\star \star}$ & & \\
\hline $\begin{array}{l}\text { Vitamin A } \\
\text { /TC } \\
\text { (ug/mmol) }\end{array}$ & $-0.198^{\star}$ & -0.073 & 0.138 & -0.065 & 0.057 & $0.194^{*}$ & $-0.218^{*}$ & -0.098 & 0.084 & $0.617^{\star \star}$ & $0.541^{\star *}$ & \\
\hline
\end{tabular}

a Spearman rank correlation coefficient. ${ }^{* *}$ Significant correlation at $p<0.01$ (two-tailed). ${ }^{*}$ Significant correlation at $p<0.05$ (two-tailed).

FSH, follicle-stimulating hormone; LH, uteinizing hormone; E2, estradiol; AMH, anti-Müllerian hormone

Table 4 gave the associations of TC-adjusted vitamin A with POI risk that determined by using binary logistic regression. The unadjusted odds ratio (OR) and $95 \%$ confidence interval $(\mathrm{Cl})$ were 0.988 and $0.977-0.999$ respectively, and the association was significant $(p=0.039)$. After adjustment for age, BMI, annual household income, and education, the same trend was found (OR $=0.986,95 \% \mathrm{Cl}: 0.972-0.999, p=0.040)$.

Table 4

Association of vitamin A with POI in binary logistic regression models

\begin{tabular}{|c|c|c|c|c|}
\hline \multirow[t]{2}{*}{ Compound } & \multicolumn{2}{|l|}{ Unadjusted Model } & \multicolumn{2}{|c|}{ Adjusted Model a } \\
\hline & OR (95\% Cl) & $\mathrm{p}$-Value & OR $(95 \% \mathrm{Cl})$ & $\mathrm{p}$-Value \\
\hline Vitamin AVTC & $N=114$ & & $N=114$ & \\
\hline (ug/mmol) & $0.988(0.977-0.999)$ & 0.039 & $0.986(0.972-0.999)$ & 0.040 \\
\hline
\end{tabular}

\section{Discussion}

Vitamin A is crucial to the human body, and the stability of vitamin A levels is essential for female reproduction [19], such as follicular development [9], ovarian steroidogenesis [11], oocyte maturation [12], and corpus luteum formation [20]. In this research, we found that serum level of vitamin A/TC is lower in POI than normal people, and vitamin A/TC was negatively associated with POI risk, indicating that vitamin A deficiency may be a risk factor for POI development. 
$\mathrm{POI}$ has an incidence of approximately $1 \%$ in women and increase with years [1]. In this study, the mean age of POI patients were $33.30 \pm 6.45$ years, the youngest was only 18 years old, it is a rather young age that POI will affect a longtime life. We observed that women in the control group showed a significantly higher annual household income and education than those in the POI group (both $p<0.001$ ). Similar results were found in a study in India, which reported that rural women with a low household income, nutritional deficiencies, and low educational attainment showed a higher risk of POI [21]. Health education should be incorporated in the health care system to tackle the health problems associated with POI.

We focus on the association of vitamin A and POI. According to the test system specification, in this study, the normal reference range of serum vitamin A was $300-1200 \mu \mathrm{g} / \mathrm{L}$. Serum vitamin A levels in the POI and control groups were $728.00 \pm 176.00$ and $503.93 \pm 145.64 \mu \mathrm{g} / \mathrm{L}$, respectively. No participant in our study suffered from VAD or excessive vitamin A levels. In our study, POI patients showed higher serum vitamin A and cholesterol levels; however, the control group showed lower serum vitamin A and cholesterol levels. Vitamin A is a fat-soluble vitamin, and its plasma levels changes with synchronized lipid levels. We use vitamin A/TC ratio to eliminate the confounding effects of lipids in evaluating the effect of vitamin A in POI. It could be seen that the serum vitamin A levels in the POI was a little bit higher, but after adjust with serum TC levels, the serum vitamin A/TC ratio was significantly lower in the women with POI than that in controls $(143.14 \pm 35.86$ vs $157.56 \pm 35.21 \mu \mathrm{g} / \mathrm{mmol}, p=0.035)$. It is believed that serum FSH level of 40 IU/L could be a maker of late-stage POI [22]. In this study, we see that the serum vitamin A/TC levels in the POI group with FSH levels $<40 \mathrm{IU} / \mathrm{L}$ were higher than those in the POI group with FSH levels $\geq 40$ IU/L. Increased serum FSH levels are associated with reduced vitamin A levels. Thus, our results seems suggest that vitamin A decrease with the POI stage increasing.

Furthermore, annual household income, education, serum AMH levels, and serum vitamin A/TC ratio were all significantly and negatively associated with POI risk. Binary logistic regression analysis shows the associations between TC-adjusted vitamin A levels and POI risk (OR $=0.9988,95 \% \mathrm{Cl}$ : $0.977-0.999, p=$ 0.039). After adjusting for annual household income, serum $\mathrm{AMH}$ levels, and education, the same trend was noted $(\mathrm{OR}=0.986,95 \% \mathrm{Cl}$ : $0.972-0.999, p=$

0.040). These results suggest that serum vitamin A/TC ratio is a predictor of POI incidence. Vitamin A may be a protective factor in ovarian tissue. Kazami et al. found that dietary antioxidant vitamins intake improving the oocyte competence [23]. Therefore, we speculate that increasing the intake of vitamin A-rich foods or using vitamin A nutritional supplements can help prevent and treat $\mathrm{POI}$.

A limitation of our study is the relatively small size of the study groups, although the results of this study are encouraging. Because of the relatively small number of cases, further studies with a large number of patients are necessary to validate these findings. Second, our study aimed to determine whether serum vitamin A levels we detected can reflect ovarian vitamin A levels. Vitamin A levels in ovarian tissues can likely be detected directly to evaluate the correlation between vitamin A and ovarian function in the future.

In conclusion, our study revealed a negative association between corrected serum vitamin A levels and POI risk. Serum vitamin A levels were a protective factor for POI, and adequate vitamin A supplementation may help prevent or delay POI development. Nonetheless, the exact etiology warrants further research.

\section{Declarations}

\section{Acknowledgments}

We thank all women who participated in this study.

\section{Author contributions}

Y.S. and P.Q.C. data analyses, manuscipt writing and editing. W.X.X. data collection and data analyses. Y.Z.H., Y.X.J., C.M.L., Y.B.L., K.T.C. data collection and clinical consultation. L.J.M. and J.H.Z. manuscript revision.

\section{Fundings}

This work was supported by the National Natural Science Foundation of China $(81701368,82003469,81801405,81873810)$, Plan for Key Research and development of Zhejiang (2018C03G2011).

\section{Compliance with ethical standards}

\section{Conflicts of interest}

The authors report no conflict of interest. The authors alone are responsible for the content and writing of this paper.

\section{Ethical approval}

The study was approved by the Human Ethics Committee of Women's Hospital, Zhejiang University School of Medicine (No. 20150021).

\section{Informed consent}


Informed consent was obtained from every participant at the initial interview.

\section{References}

1. Rudnicka E, Kruszewska J, Klicka K et al (2018) Premature ovarian insufficiency - aetiopathology, epidemiology, and diagnostic evaluation. Menopausal Review 17(3):105-108

2. Sophia T, Nick P, John CS (2019) Premature Ovarian Insufficiency and Long-Term Health Consequences. Curr Vasc Pharmacol 17(6):604-609

3. Gunning MN, Meun C, Rijn BBV et al (2020) The cardiovascular risk profile of middle age women previously diagnosed with premature ovarian insufficiency: A case-control study. PLoS ONE 15(3):e0229576

4. Nilsson E, Klukovich R, Sadler-Riggleman I et al (2018) Environmental toxicant induced epigenetic transgenerational inheritance of ovarian pathology and granulosa cell epigenome and transcriptome alterations: ancestral origins of polycystic ovarian syndrome and primary ovarian insufiency. Epigenetics 13(8):875-895

5. Simpson JL (2008) Genetic and phenotypic heterogeneity in ovarian failure: overview of selected candidate genes. Ann N Y Acad Sci 1135:146-154

6. Li C, Cao M, Ma L et al (2018) Pyrethroid Pesticide Exposure and Risk of Primary Ovarian Insufficiency in Chinese Women. Environ Sci Technol 52(5):3240-3248

7. Ward SJ, Morriss-Kay GM (1997) The functional basis of tissue-specific retinoic acid signaling in embryos. Semin Cell Dev Biol 8(4):429-435

8. Hui L, Margaret CD (2009) Vitamin A deficiency blocks the initiation of meiosis of germ cells in the developing rat ovary in vivo. Biol Reprod 81(5):9961001

9. Kawai T, Yanaka N, Richards JS et al (2016) De Novo-Synthesized Retinoic Acid in Ovarian Antral Follicles Enhances FSH-Mediated Ovarian Follicular Cell Differentiation and Female Fertility. Endocrinology 157(5):2160-2172

10. Abdelnour SA, El-Hack MEA, Swelum AA et al (2019) The Usefulness of Retinoic Acid Supplementation during In Vitro Oocyte Maturation for the In Vitro Embryo Production of Livestock: A Review. Animals (Basel) 9(8):561

11. Endo M, Nicholls, et al (2019) Retinoic Acid and Germ Cell Development in the Ovary and Testis. Biomolecules 9(12):775

12. Tahaei LS, Eimani H, Yazdi PE et al (2011) Effects of retinoic acid on maturation of immature mouse oocytes in the presence and absence of a granulosa cell co-culture system. J Assist Reprod Genet 28(6):553-558

13. Patrycja Skowrońska, Kunicki M, Pastuszek E et al (2020) Follicular fat-soluble vitamins as markers of oocyte competency. Syst Biol Reprod Med 66(2):112-121

14. Bailey RL, West KP Jr, Black RE (2015) The epidemiology of global micronutrient deficiencies. Ann Nutr Metab 66(Suppl 2):22-33

15. Webber L, Davies M, Anderson R et al (2016) ESHRE Guideline: management of women with premature ovarian insufficiency. Hum Reprod 31(5):926-937

16. Jordan P, Brubacher D, Moser U et al (1995) Vitamin E and vitamin A concentrations in plasma adjusted for cholesterol and triglycerides by multiple regression. Clin Chem 41(6 Pt 1):924-927

17. Horwitt MK, Harvey CC, Dahm CH et al (1972) Relationship between tocopherol and serum lipid levels for determination of nutritional adequacy. Ann $\mathrm{N} Y$ Acad Sci 203:223-236

18. Thurnham DI, Davies JA, Crump BJ et al (1986) The use of different lipids to express serum tocopherol: lipid ratios for the measurement of vitamin E status. Ann Clin Biochem 23(Pt 5):514-520

19. Clagett-Dame M, Knutson D (2011) Vitamin A in reproduction and development. Nutrients 3(4):385-428

20. Clagett-Dame M, Deluca HF (2002) The role of vitamin A in mammalian reproduction and embryonic development. Annu Rev Nutr 22:347-381

21. Jungari SB, Chauhan BG (2017) Prevalence and Determinants of Premature Menopause among Indian Women: Issues and Challenges Ahead. Health Soc Work 42(2):79-86

22. Harlow SD, Mitchell ES, Crawford S et al (2008) The ReSTAGE Collaboration: defining optimal bleeding criteria for onset of early menopausal transition. Fertil Steril 89(1):129-140

23. Kazemi A, Ramezanzadeh F, Nasr-Esfahani MH (2015) The relations between dietary antioxidant vitamins intake and oxidative stress in follicular fluid and ART outcomes. Iran J Reprod Med 13(9):533-540 